\title{
Erratum to: A segregation distortion locus located on linkage group 4 of the chickpea genetic map
}

\author{
P. Castro · J. Rubio $\cdot$ A. Cabrera • \\ T. Millán · J. Gil
}

Published online: 2 March 2011

(C) Springer Science+Business Media B.V. 2011

\section{Erratum to: Euphytica \\ DOI 10.1007/s10681-011-0356-7}

Due to an unfortunate mistake in the production process, part of the heading of Table 2 is missing in the above mentioned publication. The correct representation of Table 2 is published below and should be treated as definitive by the reader:

Table 2 Chi-square analysis of non-Mendelian segregation under three different hypotheses $\left(\chi_{(p=q)}^{2} ; \chi_{\left(p^{2}: 2 p q: q^{2}\right)}^{2} ; \chi_{(0.5 p: 0.5: 0.5 q)}^{2}\right)$ in a chickpea $F_{2}$ population $\left(\mathrm{F}_{2}-1\right)$ derived from the intraspecific cross ILC3279 $\times$ WR315

\begin{tabular}{|c|c|c|c|c|c|c|c|}
\hline \multirow[t]{3}{*}{ Marker } & \multicolumn{4}{|c|}{ Gametic selection in male and female } & \multicolumn{3}{|c|}{ Gametic selection in male or female } \\
\hline & \multicolumn{2}{|c|}{ Allelic frequency } & \multicolumn{2}{|l|}{$\chi_{1}^{2}$} & \multicolumn{2}{|c|}{ Allelic frequency } & \multirow{2}{*}{$\frac{\chi_{1}^{2}}{0.5 p: 0.5: 0.5 q}$} \\
\hline & $p$ & $q$ & $p=q$ & $p^{2}: 2 p q: q^{2}$ & $p$ & $q$ & \\
\hline GAA47 & 0.405 & 0.595 & $42.45^{* * *}$ & 0.00 & 0.315 & 0.684 & 0.68 \\
\hline TA72 & 0.285 & 0.715 & $215.59 * * *$ & $35.37 * * *$ & 0.062 & 0.937 & 0.14 \\
\hline SC-Y17 & 0.274 & 0.726 & $235.76^{* * *}$ & $34.99 * * *$ & 0.051 & 0.948 & 0.04 \\
\hline
\end{tabular}

*, ** and *** are significant at $P<0.05, P<0.01$ and $P<0.001$ levels, respectively

The online version of the original article can be found under doi:10.1007/s10681-011-0356-7.

P. Castro $(\bowtie) \cdot$ J. Rubio

Área de Mejora y Biotecnología, IFAPA, Centro

'Alameda del Obispo', Apdo. 3092,

14080 Córdoba, Spain

e-mail: patriciar.castro@juntadeandalucia.es

A. Cabrera · T. Millán · J. Gil

Dpto Genética, Universidad de Córdoba, Campus de,

Rabanales Edificio C5 2a planta, 14071 Córdoba, Spain 